\section{Common mental disorders in primary care}

\author{
Essays in honour of Professor \\ David Goldberg
}

edited by Michele Tansella \& Graham Thornicroft. Published by Routledge, London and New York, 1999, 272 pages,

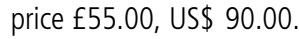

One of the few issues on which health care planners agree is that primary and general health care services should be responsible for the treatment of common mental disorders such as anxiety and depression. The high prevalences of these disorders and the frequency with which sufferers seek help from the general medical services — argue against a specialist response to their needs for treatment. Though currently these disorders often fail to be diagnosed, to the detriment of patients, their families and the wider community, research in both industrialized and developing countries has shown that it is possible to train primary health workers to identify them and to treat them effectively.

The stakes are high. By the year 2020, it is estimated that depression, a common mental disorder, will constitute the second greatest disease burden in the world, as measured by disability-adjusted life years. In addition to the suffering of individuals and their families, there is also a heavy economic burden for all concerned and for countries. The need for mainstream services to deal with such common mental disorders raises the question of how to provide quality care to sufferers. This question is pertinent now but was perhaps even more so in the mid-1960s, when Professor Sir David Goldberg began his work in this field. The publication of this book marks his retirement from the Institute of Psychiatry in London.

The distinguished contributors to the book bring together clinical and research work drawn from psychiatry, medicine, psychology, social work and sociology, covering such topics as: the biological basis of common mental disorders; disability and depression in primary care; the limits of mental health care in general medical clinics; improving the management of mental disorders in the elderly; and training the whole primary care team. Common mental disorders in primary care is neither a basic primer nor a comprehensive review. Rather, it is at once a vast storehouse of knowledge and a treasury of gems of perception and description.

One such gem is the section on teamwork, starting with a detailed account of rural mental health services - the Gujarkhan demonstration project of Pakistan's national mental health plan from which the reader learns that there is one qualified psychiatrist for every million people, that allied professionals such as psychiatric nurses, psychologists and social workers are few and far between, and that $66 \%$ of the total population are not within reach of the services. However, one also learns that, despite these limitations, a well-thought-out policy that harnesses local resources can reap significant gains in mental well-being. This account is followed by a chapter calling for social care to be developed in primary care (based mainly on literature from the United Kingdom). The section ends with a discussion of the future role of the nurse in primary care and how nurses will come to terms with the advent of high technology computer-assisted treatment.

The contrast in the situations described in these chapters puts the situation of poorer countries in sharp relief, but one wonders how much the industrialized world can learn from the breadth of interventions offered by the Gujarkhan project. Certainly, if the training of primary health care workers, schoolteachers and community leaders can be maintained, and if intersectoral collaboration and the community mental health programme can be further developed, Gujarkhan will have effectively mobilized indigenous resources and decreased the stigma of mental illness in a way that many industrialized countries would envy. The project will have developed an innovative base from which mainstream services can be launched.

The rest of the book is equally diverse, ranging from policy analyses through social theory to experimental neurobiology. It covers management issues, disorders of old age, computerized assessments, guidelines for diagnosis, training packages, the limits of psychiatry, funding and conceptual modelling, as well as the basic epidemiology of common mental disorders.

The only criticism is that the book could have had more input from the non-industrialized world and could have included more analyses of the impact of social policy on the prevalence of common mental illnesses. However, while not attempting to be comprehensive, these essays provide a wealth of information that most readers will find both useful and stimulating.

\section{Kwame McKenzie}

Institute of Psychiatry

De Gespigny Park

London SE5 8AF

England

(e-mail: sphakjm@iop.kcl.ac.uk) 